\title{
PROGRESIÓN RÁPIDA DE SÍNDROME DE STEVENS-JOHNSON A NECRÓLISIS EPIDÉRMICA TÓXICA SECUNDARIO A LAMOTRIGINA
}

\section{QUICK PROGRESSION OF STEVENS-JOHNSON SYNDROME TO TOXIC EPIDERMAL NECROLYSIS SECONDARY TO LAMOTRIGINE}

\author{
Núñez González, Ivana*; Stanziola Paredes, Franshesca*; Benedito Reina, Vielka* \\ *Médico interno de primera categoría, Hospital Regional de Azuero “Anita Moreno", La Villa de los Santos, Panamá \\ Recibido: 2 de diciembre del 2019 \\ Aceptado: 17 de mayo del 2020 \\ Núñez I, Stanziola F, Benedito V. Progresión Rápida de Síndrome de Stevens-Johnson a Necrólisis Epidérmica Tóxica Secundario a Lamotrigina. \\ Reporte de un caso. Rev méd cient. 2020;33:8-15. DOI: 10.37416/rmc.v33i1.538
}

\section{RESUMEN}

Masculino de 19 años procedente de la provincia de Los Santos, Panamá; en estudio por convulsiones de tipo generalizado por lo que recibió lamotrigina durante tres semanas, acude con historia de siete días de evolución de malestar general, adinamia, fiebre no graduada sin predominio horario, conjuntivitis bilateral y desarrollo progresivo de un eritema generalizado y máculas purpúricas con predominio inicial en el área facial, cuello, tronco y el área proximal de las extremidades superiores. Además de prurito generalizado y sensibilidad cutánea. Durante la hospitalización ocurre diseminación de lesiones a toda la superficie corporal y aparición de ampollas de contenido claro, áreas de desprendimiento epidérmico y afectación de mucosas, que abarcó al inicio menos del $10 \%$ de la superficie corporal total con progresión rápida a más del $80 \%$ en menos de 24 horas. Fue trasladado a Unidad de Cuidados Intensivos en donde recibió antibióticos sistémicos, corticoides e inmunoglobulina endovenosa con evolución favorable.

PALABRAS CLAVE: Síndrome de Stevens-Johnson, Necrólisis epidérmica tóxica, lamotrigina.

\section{ABSTRACT}

A 19 year old male from the province of Los Santos, Panama; under study for generalized seizures for which he received lamotrigine during three weeks, presented with a seven-day history of evolution of general malaise, adynamia, unregulated fever without hourly predominance, bilateral conjunctivitis and progressive development of generalized erythema and purpuric macules, with initial predominance on the face, neck, trunk and proximal upper extremities in addition to generalized pruritus and skin sensitivity. During hospitalization the lesions disseminated to the rest of the body surface accompanied by blisters with clear content, areas of epidermal detachment and mucosal involvement, which initially covered less than $10 \%$ of the total body surface area with rapid progression to more than $80 \%$ in less than 24 hours. He was transferred to the Intensive Care Unit where he received systemic antibiotics, corticosteroids, and intravenous immunoglobulin with a favorable outcome.

KEYWORDS: Stevens - Johnson syndrome, Toxic Epidermal Necrolysis, Lamotrigine.

Progresión Rápida de Síndrome de Stevens-Johnson a Necrólisis Epidérmica Tóxica Secundario a Lamotrigina.

Reporte de un caso by Núñez I, Stanziola F, Benedito V is licensed under a Creative Commons Attribution-

NonCommercial-NoDerivs 4.0 Unported License.

Permissions beyond the scope of this license may be available at www.revistamedicocientifica.org. 


\section{INTRODUCCIÓN}

El síndrome de Stevens-Johnson (SSJ) y la necrólisis epidermolítica tóxica (NET) constituyen parte del espectro grave de las reacciones cutáneas adversas severas que afortunadamente no son muy comunes y tienen una baja incidencia de acuerdo con las estadísticas que existen actualmente.

En Panamá existen pocos casos reportados de estos eventos, pero son patologías que deben tenerse en cuenta ya que conllevan una alta tasa de morbilidad y mortalidad. Es un diagnóstico que debe tenerse presente ante la historia clínica exhaustiva sobre todo acerca de la ingesta de medicamentos que constituye su etiología principal en la mayoría de los casos.

En este caso clínico se presenta el cuadro desarrollado por un joven a partir del uso de medicamentos anticonvulsivantes, posterior tratamiento y evolución.

\section{CASO CLÍNICO}

\section{ENFERMEDAD ACTUAL}

Paciente masculino de 19 años de edad procedente de la provincia de Los Santos con antecedente personal patológico de hipoacusia derecha y en estudio por convulsiones de tipo generalizadas por lo cual estuvo en tratamiento durante tres semanas con lamotrigina, que acude con cuadro de aproximadamente siete días de evolución caracterizado por malestar general, astenia, adinamia, fiebre no graduada sin predominio horario y conjuntivitis bilateral, asociado a la aparición gradual de eritema generalizado y máculas purpúricas predominantes inicialmente en área facial, cuello, tronco y extremidades superiores a nivel proximal, además de prurito generalizado y sensibilidad cutánea. Durante su primer día de hospitalización cursa con diseminación de las lesiones al resto de la superficie corporal junto con aparición de ampollas de contenido claro, áreas de desprendimiento epidérmico y afectación de mucosas, con afección inicial del 10\% de la superficie corporal que progresó rápidamente a más del $80 \%$ en menos de 24 horas.

Como antecedentes personales presenta hipoacusia derecha diagnosticada en la infancia y aún en seguimiento; convulsiones de tipo generalizadas con inicio aproximado hace 7 a 8 meses, por lo que fue medicado por Neurología con lamotrigina tres semanas previas. No refiere otras enfermedades crónicas, alergias, transfusiones de hemoderivados o traumatismos previos.

No refiere antecedentes quirúrgicos.

Consume alcohol tipo whisky de 3-4 veces al mes sin llegar a la embriaguez. No fuma ni hace uso de drogas ilícitas.

No declara antecedente heredofamiliar de importancia.

\section{EXAMEN FÍSICO}

- Signos vitales: Presión arterial: 115/65 mm Hg; Frecuencia cardíaca: 114 latidos/minuto; Frecuencia respiratoria: 16 respiraciones/minuto; Temperatura: 37,0 ㄷ; Saturación periférica de oxígeno (oximetría de pulso): $96 \%$.

- Hiperemia conjuntival, secreción purulenta, fotofobia, pupilas normoreactivas a la luz. (Ver Figura 1 y 2).

- Apertura oral limitada; mucosa oral subhidratada, eritematosa, con higiene regular y dolor a la manipulación.

- Cuello y tórax simétrico, patrón respiratorio normal, eritema generalizado en tronco superior con calor a la palpación, máculas coalescentes en tronco inferior, ampollas de contenido claro en tórax antero-posterior y áreas localizadas de desprendimiento epidérmico. (Ver figura 3, 4 y 5).

- Ruidos cardiacos rítmicos regulares, sin soplos.

- Ruidos respiratorios presentes y normales en ambos campos; no se auscultan ruidos agregados.

- Abdomen plano, blando y depresible. Ruidos hidroaéreos presentes, no doloroso a la palpación, no se palpan visceromegalias. Ampollas en área periumbilical.

- Extremidades simétricas, eritema generalizado predominante a nivel proximal en miembros superiores; sin limitación funcional.

Tabla 1. Laboratorios durante hospitalización

\begin{tabular}{|c|c|c|c|c|c|c|c|c|c|c|c|c|c|}
\hline Fecha & $\mathrm{Hb}$ & Heto & Leu & Neut\% & Plq & $\mathrm{Cr}$ & BUN & $\mathrm{Na}^{+}$ & $\mathrm{K}^{+}$ & $\mathrm{Ca}^{2+}$ & $\mathrm{Mg}^{2+}$ & Glc & Procalcitonina \\
\hline $18 / 10 / 18$ & 13,9 & 40,5 & 4900 & 83.0 & 227000 & 0,81 & 12,0 & 142 & 4.0 & 8,4 & & 141 & 0.28 \\
\hline $21 / 10 / 18$ & 12.5 & 35.9 & 2700 & 55.7 & 287000 & 0.56 & 18 & 144 & 4.6 & - & - & 102 & - \\
\hline $8 / 11 / 18$ & 11.7 & 33.7 & 12800 & 77.2 & 505000 & 0.58 & 18 & 145 & 4.6 & 11.3 & 2.0 & 116 & 0.07 \\
\hline $12 / 11 / 18$ & 12.0 & 34.2 & 7400 & 56 & 448000 & 0.55 & 18 & 144 & 4.8 & 11.2 & - & 105 & $<0.05$ \\
\hline
\end{tabular}

Fuente: Laboratorio Clínico del Hospital Regional de Azuero Anita Moreno 


\section{LABORATORIOS}

23/10/18 Hemocultivo negativo a los 5 días. Muestra: catéter venoso central.

27/10/18 Coprocultivo negativo por Salmonella y Shigella. 28/10/18 Urocultivo negativo a las 48 horas de incubación. 2/11/18 Urocultivo negativo a las 48 horas de incubación.

\section{TRATAMIENTO}

El manejo de este espectro de enfermedades debe estar enfocado a la identificación de la causa y en caso de ser una sustancia o medicamento proceder a su discontinuación y luego proceder a la estabilización del paciente y minimizar la extensión del daño en la piel y mucosas para a su vez disminuir el riesgo de presentar secuelas y muerte. En esta patología es fundamental que el manejo sea de tipo multidisciplinario por la magnitud de la afección sistémica. Este paciente fue manejado inicialmente con los antibióticos endovenosos oxacilina y azitromicina; recibió también corticosteroides orales y endovenosos e inmunoglobulina endovenosa; posteriormente trasladado a Unidad de Cuidados Intensivos donde estuvo hospitalizado durante 33 días recibiendo manejo conjunto con Oftalmología por úlcera corneal. Fue manejado como un gran quemado con curaciones con solución salina, clorhexidina y apósitos epitelizantes con zinc y plata, además de matriz a base de submucosa intestinal de porcino en la cara. En cuanto al manejo ocular, recibió terapia antibiótica oftálmica tópica y corticoesteroidea.

Figura 1. Erosiones en los párpados superiores e inferiores recubiertas por costras hemáticas y afectación de la mucosa oral (exfoliación y mucositis).

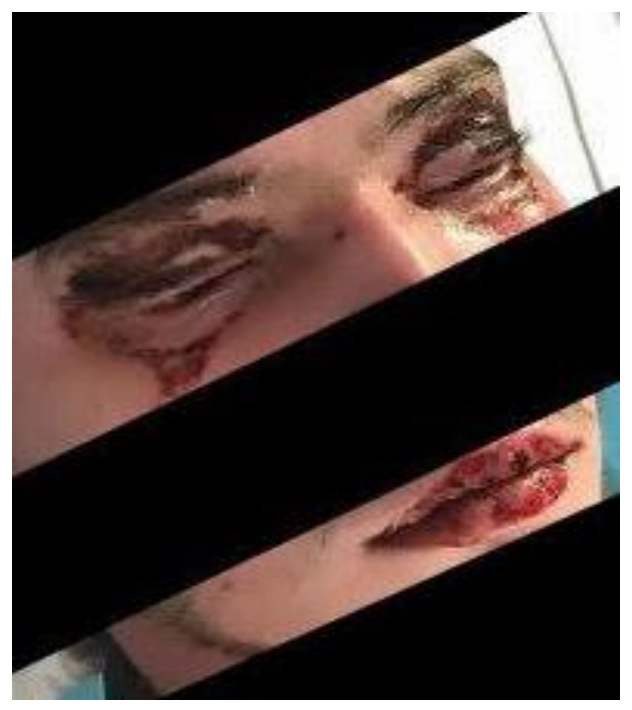

Figura 2. Uveítis, blefaritis, meibomitis y úlcera corneal inferior secundario a síndrome de ojo seco.



Figura 3. Imágenes clínicas de eritema multiforme mayor.

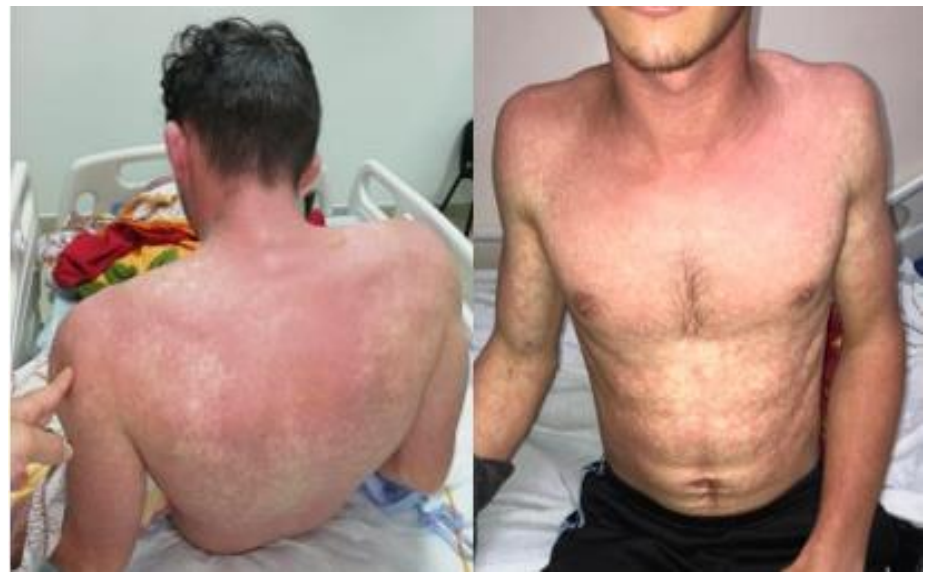

Se observan pápulas aisladas y otras que confluyen en placas eritematosas diseminadas, típicas lesiones en diana (lesiones elevadas con halo rosado separado por un anillo pálido) en tórax posterior, tórax anterior, abdomen y miembros superiores. 
Figura 4. Placas eritematosas con aparición de lesiones vesiculo-ampollares de contenido claro en tórax posterior.

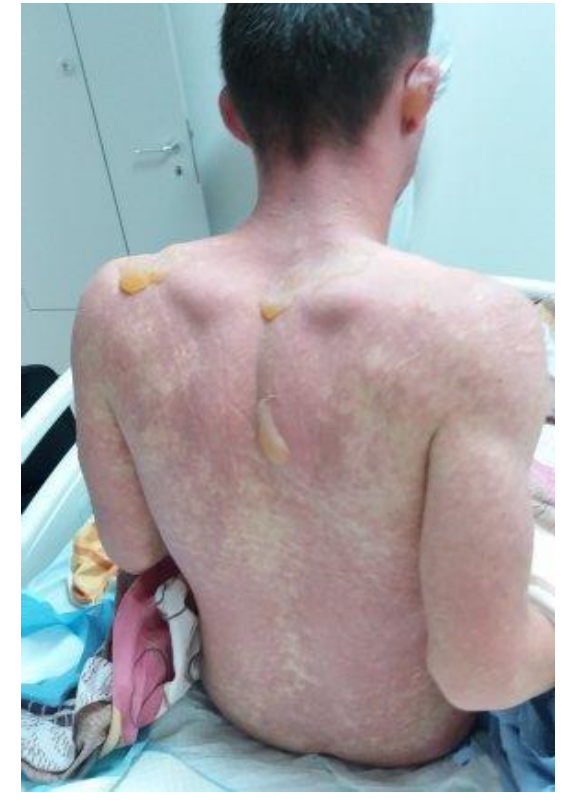

Figura 5. Necrólisis epidérmolítica tóxica.

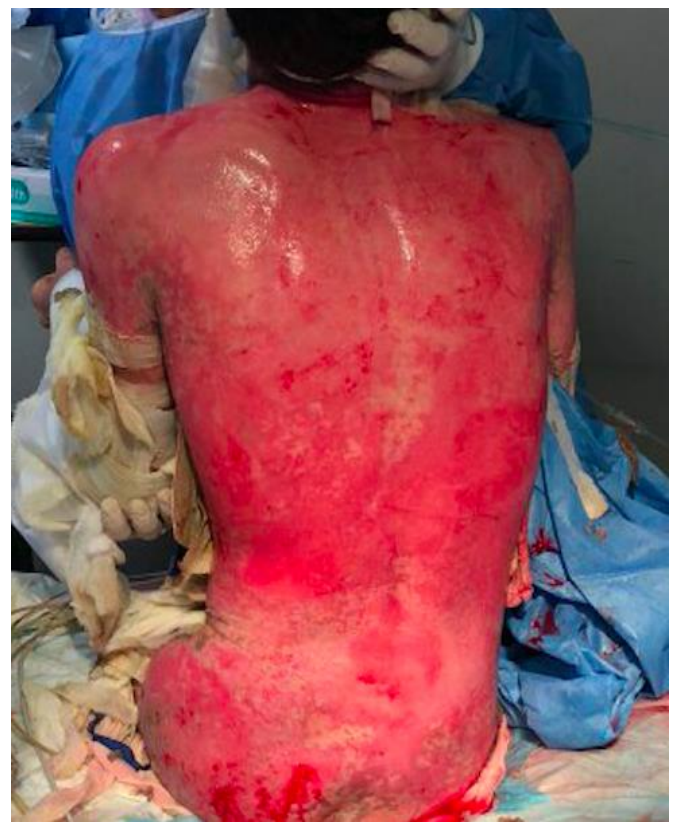

Se observa desprendimiento de la epidermis generalizado (espalda, miembros superiores, glúteos).

\section{DIAGNÓSTICO DIFERENCIAL}

Existe una gran variedad de diagnósticos diferenciales para esta entidad. Se mencionan ejemplos a continuación y sus diferencias con el caso presentado anteriormente.

\section{Eritema multiforme mayor}

Este se acompaña frecuentemente de un cuadro febril y en ocasiones con artralgias; en este caso no fue evidenciado, pero podría ser también una manifestación clínica. Las lesiones se centran principalmente en la cara y extremidades, pero se observan pápulas de color eritematoso a rojo oscuro en el tronco con mayor frecuencia que en el eritema multiforme menor. Afecta la mucosa oral y los labios con desprendimiento hemorrágico; las áreas genitales y ocular pueden estar involucradas, pero es menos frecuente; en el caso de nuestro paciente esto está severamente afectado. Cuando existe la presencia de ampollas, el eritema multiforme mayor se distingue del SSJ, morfológicamente por la presencia de púrpura o ampollas en lesiones maculares del tronco. ${ }^{1}$

\section{Pénfigo vulgar}

Se caracteriza por ampollas grandes, dolorosas que se rompen fácilmente para formar erosiones en piel y membranas mucosas; el líquido en la ampolla es claro, pero puede volverse seropurulento o hemorrágico. Posteriormente se forman costras y cuando finalmente sanan se observan máculas hiperpigmentados sin cicatrices. Por lo general aparece primero en la boca o sitio de una quemadura. El signo de Nikolsky es positivo al igual que ocurre en el espectro de SSJ/NET. El diagnóstico se realiza por biopsia y estudios inmunes. ${ }^{1,2} \mathrm{EI}$ cuadro de nuestro paciente inició con eritema generalizado y máculas purpúricas predominantes inicialmente en área facial, cuello, tronco y extremidades superiores a nivel proximal además de prurito generalizado y sensibilidad cutánea. Durante su primer día de hospitalización cursa con diseminación de las lesiones al resto de la superficie corporal junto con aparición de ampollas de contenido claro, áreas de desprendimiento epidérmico y afectación de mucosas.

\section{Penfigoide de membranas mucosas}

En esta patología las membranas mucosas se ven generalmente afectadas (cavidad oral, conjuntiva, piel) con vesículas dolorosas que se rompen rápidamente, y existen complicaciones como discapacidad visual hasta ceguera total. La afectación única de la mucosa oral puede durar años. La salud general del paciente usualmente no se compromete, llegando a ser una enfermedad crónica. El diagnóstico es clínico y mediante biopsia. ${ }^{1,2}$ En nuestro caso hubo afectación de la mayoría de la superficie corporal en menos de 24 horas con compromiso importante del estado general del paciente. 


\section{Penfigoide ampolloso}

Es la enfermedad autoinmunitaria ampollar más frecuente, afectando en mayor número a personas de edad avanzada pero también se ven casos en jóvenes. Inicia con un cuadro de prurito, luego lesiones eccematosas y urticarianas. Posteriormente desarrollan ampollas grandes y tensas de contenido claro seroso en distribución generalizada asociado a vesículas. Además de las ampollas se observan placas con un patrón anular. La afectación a las mucosas es rara. El eritema puede permanecer semanas o meses. ${ }^{1,2}$ El signo de Nikolsky es negativo. En nuestro caso, el paciente es joven y presenta gran afectación de mucosas además de estar presente el signo de Nikolsky.

\section{Pénfigo paraneoplásico}

Se observan lesiones cutáneas (máculas eritematosas, liquenoides, eritema multiforme, ampollas) y en todos los casos hay lesiones orales, las cuales pueden ser la única manifestación; esta es grave y progresiva. También puede presentarse con afectación ocular. La mayoría de los pacientes presentan neoplasias asociadas. 1,2 El paciente presentó estas lesiones cutáneas, junto con la afectación ocular y oral, sin neoplasia asociada ni afectación pulmonar, el cual mejoró con curaciones como un gran quemado, inmunoglobulina y corticosteroides.

\section{Lupus eritematoso ampolloso}

Se presenta como vesículas o ampollas individuales o agrupadas con mayor frecuencia en áreas expuestas al sol, pero pueden ser generalizadas; en raras ocasiones producen prurito. Esta enfermedad presenta lesión cutánea, articular y visceral y no se relaciona a medicamentos, sino a factores genéticos, ambientales y hormonales. ${ }^{1,2}$ El paciente desarrolló el cuadro secundario a lamotrigina y cursó con una buena evolución con tratamiento sin afectar a nivel visceral, ni articular.

\section{Pustulosis exantemática aguda generalizada}

Se desencadena generalmente por fármacos (ej.: antibióticos del grupo betalactámicos). Inicia con una erupción eritematosa con múltiples pústulas pequeñas afectando el estado general del paciente y en los exámenes complementarios se presenta con leucocitosis, eosinofilia e insuficiencia renal, incluso falla hepática; pocas veces afecta mucosas a diferencia de lo que ocurre en este caso. El diagnóstico es clínico además de estudios inmunohistológicos específicos que confirman la reacción específica mediada por los linfocitos T. ${ }^{1,2}$

\section{Síndrome de hipersensibilidad inducida (SHDI)/ reacción medicamentosa con eosinofilia y síntomas sistémicos (síndrome de DRESS)}

Constituye una erupción cutánea severa con afectación sistémica que involucra activación de las células T con posterior reacción inflamatoria en múltiples órganos. ${ }^{1}$ Se caracteriza por rash, fiebre, adenopatías y compromiso sistémico además de leucocitosis o linfocitosis atípica. ${ }^{8}$ La erupción cutánea se desarrolla de manera tardía (usualmente después de dos semanas luego de haber tenido exposición al medicamento desencadenante). Los síntomas son duraderos y persisten incluso después de haber retirado el agente causal. Puede ocurrir con medicamentos de casi cualquier categoría, aunque se presenta con más frecuencia debido a la exposición a ciertos medicamentos. La erupción que caracteriza a este síndrome es de tipo morbiliforme y puede presentarse desde formas leves hasta severas con eritrodermia exfoliativa. La mucositis es poco común, pero puede ocurrir también principalmente en el área bucal y de forma más leve que en el Síndrome de StevensJohnson. No se requiere de biopsia de piel para confirmar este diagnóstico. ${ }^{1}$

\section{REVISIÓN BIBLIOGRÁFICA}

\section{DEFINICIÓN}

La necrólisis epidérmica tóxica es una reacción mucocutánea grave que resulta de una respuesta inmunológica inadecuada debida a la exposición a varios medicamentos y con menor frecuencia a infecciones por hongos, virus causando el desprendimiento de la epidermis con afectación simultánea de las mucosas. La necrólisis epidérmica tóxica está estrechamente relacionada con el síndrome de StevensJohnson, diferenciándose en el porcentaje de superficie cutánea comprometida; en donde la necrólisis epidérmica tóxica tiene afectación mayor a un $30 \%$ y el síndrome de Stevens-Johnson menor a un $10 \% .^{3,4}$ Existe una superposición entre ambas entidades (SSJ/NET) que presenta un porcentaje de superficie cutánea afectada entre el $10-30 \%{ }^{1}$

\section{EPIDEMIOLOGÍA}

La necrólisis epidérmica tóxica es la principal y más grave urgencia dermatológica, con una incidencia de 1 a 6 casos por 
millón de habitantes al año en Europa y Estados Unidos. Está principalmente relacionada al uso de fármacos tales como AINES, anticonvulsivantes y algunos antibióticos; siendo un poco más frecuente en las mujeres que en los hombres a razón de 1.7 casos. La NET está relacionada a una alta mortalidad estimada entre 30 a 40\%, mientras que el Síndrome de Stevens - Johnson presenta un índice de mortalidad que oscila en 1-5\%, siendo en ambas las principales causas de muerte la sepsis y el shock séptico. ${ }^{5,6,7}$

\section{ETIOLOGÍA}

La etiología del síndrome de Stevens-Johnson y la necrólisis epidérmica tóxica no está esclarecida del todo. Se considera un espectro de enfermedades cuya causa aún sigue en estudio y se ha planteado que se debe a múltiples causas, teniendo en cuenta el impacto de la predisposición genética en su desarrollo. ${ }^{8}$

\section{FISIOPATOLOGÍA}

Se ha evidenciado que los pacientes con predisposición genética tienen mayor tendencia o susceptibilidad a desarrollar reacciones adversas principalmente a la exposición de fármacos. La fisiopatología de la NET se puede resumir en tres conceptos básicos que están directamente involucrados:

- La pérdida extensa de la piel

- La respuesta inflamatoria sistémica

- La afectación de las mucosas

Los pacientes que cursan con NET presentan grandes pérdidas de superficie cutánea, lo que conlleva a una pérdida importante de fluidos corporales y electrolitos. Secundario a esto, el paciente puede presentar alteraciones hidroelectrolíticas que van acompañadas de insuficiencia renal aguda, hipotensión y acidosis, desencadenando un estado de hipoperfusión tisular y shock. Los pacientes en este estado requieren reanimación agresiva con líquidos y cuidados como un paciente gran quemado para evitar el alto riesgo de infección, ya que a falta de la barrera de protección fisiológica (la piel) aumenta el riesgo de colonización por microorganismos y sepsis secundaria.

En esta condición los pacientes desarrollan una respuesta inflamatoria sistémica secundaria a la liberación de citoquinas inflamatorias en el torrente sanguíneo, lo que ocasiona respuestas compensatorias en el paciente como la fiebre, leucocitosis, taquicardia y taquipnea. Cuando esta respuesta inflamatoria es exagerada en el organismo, condiciona a un estado de hipermetabolismo y baja respuesta del sistema inmunológico (leucopenia) lo que aumenta el riesgo de infección y como consecuencia final, la falla multiorgánica.

Adicional a esto, los pacientes pueden presentar afectación de las mucosas oral, ocular, faríngea y bronquial. La afectación ocular puede desencadenar síndrome de ojo seco con úlceras corneales secundarias, meibomitis y cicatrices que pueden causar ceguera permanente. La afectación orofaríngea y bronquial lleva a la acumulación de restos epiteliales que ocasionan dificultad para comer, deglutir, eliminar secreciones y atelectasias, con alto riesgo de desarrollar una insuficiencia respiratoria aguda que conlleve a la utilización de ventilación mecánica. ${ }^{7,9}$

\section{CUADRO CLÍNICO}

\section{Etapa aguda}

El cuadro inicial es inespecífico, caracterizándose por ser un proceso inflamatorio agudo y puede incluir síntomas constitucionales o un pródromo de cuadro gripal que se presenta antes de la aparición de las lesiones en la piel. ${ }^{10}$

Inicialmente las lesiones en la piel aparecen en la cara, tronco, palmas y plantas, asociado a eritema y erosiones en las mucosas. La morfología es variable, incluyendo desde máculas eritematosas o violáceas que pueden ser incluso dolorosas, que pasan a ser ampollas junto con signo de Nikolsky positivo. ${ }^{8}$

\section{Etapa crónica}

La aparición de secuelas es frecuente y puede manifestarse de distintas formas, por ejemplo: lesiones cutáneas hiperpigmentadas o hipopigmentadas, alteraciones ungueales como distrofia y manifestaciones oculares como queratinización conjuntival y lesiones corneales como úlceras hasta progresar a ceguera. ${ }^{8}$

\section{DIAGNÓSTICO DIFERENCIAL}

Los principales diagnósticos diferenciales que se deben tomar en cuenta son las enfermedades autoinmunes como la dermatosis por depósito lineal de IgA, penfigoide bulloso y el síndrome de piel escaldada estafilocócica. Otros diagnósticos a tener en cuenta son exantema agudo generalizado pustuloso y pénfigo paraneoplásico., 9 


\section{DIAGNÓSTICO}

El diagnóstico es clínico. Esto debe ser confirmado por biopsia e histopatología, presentando queratinocitos apoptóticos y desprendimiento de la epidermis. Los exámenes complementarios sirven de guía para evaluar la evolución y el pronóstico del paciente, pero no son diagnósticos en este caso. ${ }^{8}$

\section{TRATAMIENTO}

En caso de que se encuentre asociado a un fármaco, lo más importante es la discontinuación del mismo. ${ }^{9}$ El tratamiento es de soporte con aporte de líquidos, apoyo nutricional, control de infecciones y complicaciones. El manejo debe ser multidisciplinario y en muchas ocasiones los pacientes son manejados en unidades de cuidados intensivos y en unidades de quemados. Es esencial que el servicio de oftalmología sea consultado porque los pacientes presentan secuelas oculares como complicación más frecuente. ${ }^{2}$

El cuidado de la piel es esencial y se debe tener cuidado a la movilización del paciente y tratar de evitar cualquier fuente de trauma cutáneo. La piel se debe mantener limpia e irrigarla con frecuencia. Se recomienda el uso de emolientes y en casos más complejos se puede utilizar antibióticos tópicos en áreas muy afectadas con presencia de costras o que presenten cultivos positivos por microorganismos. El uso de parches reduce la pérdida de fluidos y limita la colonización por microorganismos. ${ }^{2}$

Es importante el aspecto broncopulmonar del paciente, se pueden indicar nebulizaciones y ejercicios respiratorios. En caso de hipoxemia puede requerir intubación, aspiración y ventilación mecánica. ${ }^{3}$

Otro aspecto importante es la analgesia ya que en muchas ocasiones presentan dolor crónico y a la movilización. ${ }^{2}$

Actualmente no hay evidencia que indique un tratamiento farmacológico específico. En algunos casos se utiliza tratamiento inmunosupresor como la inmunoglobulina o ciclosporina. Como se mencionó anteriormente, el factor de necrosis tumoral alfa al igual que otras citocinas, interferón $\gamma$, interleucina 8 y el óxido nítrico están presentes en las lesiones epidérmicas y cuentan con la capacidad o afinidad para unirse a receptores que inducen apoptosis celular. En uno de los estudios de cohorte realizados (RegiSCAR), se observó un beneficio en la supervivencia de los pacientes al utilizar en el tratamiento ciclosporinas y agentes anti-TNF, arrojando menor mortalidad y menor tiempo de re-epitelización. En general, existe poca evidencia en el uso de los agentes anti-TNF como tratamiento en el SSJ y NET. Hasta el momento, existen casos reportados en la literatura, que apuntan a que los anti-TNF son una terapia basada en el bloqueo selectivo del factor de necrosis tumoral alfa (TNF- $\alpha$ ), el cual juega un importante papel en la patogénesis de esta enfermedad. ${ }^{7,9}$ El tratamiento con corticoides y ciclofosfamida se encuentra en desuso. ${ }^{7}$

\section{PRONÓSTICO}

El paciente puede presentar secuelas a largo plazo tanto en la piel como en las mucosas, a nivel ocular y pulmonar. Estas secuelas tienen un impacto psicológico, social y económico importante en el paciente por lo que se hace énfasis en el manejo multidisciplinario. ${ }^{3}$

\section{CONCLUSIONES}

El síndrome de Stevens-Johnson y la necrólisis epidérmica tóxica constituyen entidades graves de la piel cuyo diagnóstico es clínico, el cual debe ser precoz para brindar un tratamiento oportuno y adecuado teniendo en cuenta la alta morbimortalidad y complicaciones probables.

\section{AGRADECIMIENTOS}

Se agradece a la Dra. Yarissa Montilla, Médico Intensivista del Hospital Regional de Azuero Anita Moreno y al Dr. Ricardo Milord Médico Internista del Hospital Joaquín Pablo Franco Sayas por sus aportes para el desarrollo de este caso clínico.

\section{REFERENCIAS}

1. James, W., Elston, D., Treat, J., Rosenbach, M., \& Neuhaus, I. (2020). Andrews's Diseases of the Skin Clinical Dermatology. Elsevier. 117 -119;141-142;162;453; 459-467

2. Griffiths, C., Barker, J., Bleiker, T., Chalmers, R., \& Creamer, D. (2016). Rook's Textbook of Dermatology. Wiley Blackwell. 110-47; 119.13 - 22; 50.10-50.29;147.21

3. Muñoz Guerrero, Félix; Orta Guerrero, Francisco. Necrólisis epidérmica tóxica, manejo en la Unidad de Quemados. Reporte de un caso. Archivos de Medicina de Urgencia de México. Vol. 3, Núm. 3, Septiembre-Diciembre 2011.

4. Charquille Salazar, Sara; Legrá Martínez, William; Matos Conde, Ana E; Matoses Ortiz, Gerardo. Necrólisis 
epidérmica tóxica. Presentación de un caso. Rev Inf Cient 2014; 85(3):499-506

5. Quintero Castro, D.; Cruzata Quintero, Y.; Durán Morgado, D. Síndrome de Stevens-Johnson y uso de corticosteroides. Universidad de Ciencias Médicas. Guantánamo, Cuba. Rev Inf Cient. 2017; 96(2):297-305

6. Su, S.-C., \& Chung, W.-H. (2013). Update on pathobiology in Stevens-Johnson syndrome and toxic epidermal necrolysis. Dermatologica Sinica, 175-180

7. Estrella-Alonso, A.; Aramburu, J.; González-Ruiz, M.; Cachafeiro, L.; Sánchez Sánchez, M.; Lorente, J. Necrolisis epidérmica tóxica: un paradigma de enfermedad crítica. Instituto de Investigación Sanitaria del Hospital Universitario de Getafe - Madrid, Spain. Rev Bras Ter Intensiva. 2017;29(4):499508

8. Torres $\mathrm{M}$, Olmos $\mathrm{E}$. Reacciones medicamentosas severas. Síndrome de Stevens-Johnson y síndrome DRESS. Acta Med Colomb 2013 abr-jun. 38(2):[aprox. 17 p.]. Disponible en: http://www.scielo.org.co/pdf/amc/v38n2/v38n2a08.pdf

9. Martínez-Cabriales, S.; Gómez-Flores, M.; y OcampoCandiani, J. Actualidades en farmacodermias severas: Síndrome de Stevens-Johnson (SSJ) y Necrólisis epidérmica tóxica (NET). Departamento de Dermatología, Hospital Universitario Dr. José Eleuterio González, Monterrey, N.L., México. Gac Med Mex. 2015;151: 777-87

10. Sotelo-Cruz N.; Síndrome de Stevens-Johnson y necrólisis epidérmica tóxica en los niños. Gaceta Médica de México. 2012; 148:265-75. Disponible en: https://www.medigraphic.com/pdfs/gaceta/gm-

2012/gm123h.pdf 